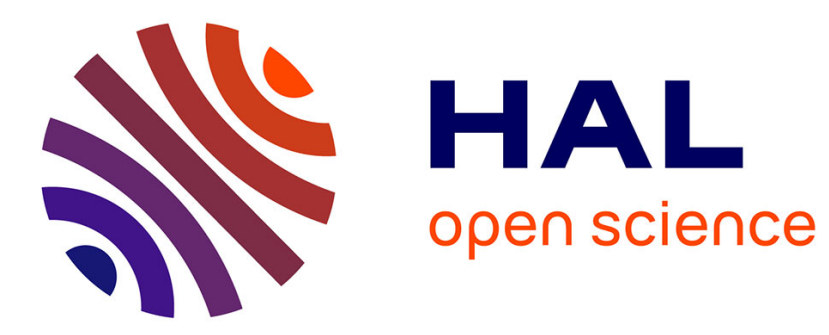

\title{
Molecular Simulation of Electrode-Solution Interfaces
}

\author{
Laura Scalfi, Mathieu Salanne, Benjamin Rotenberg
}

\section{To cite this version:}

Laura Scalfi, Mathieu Salanne, Benjamin Rotenberg. Molecular Simulation of Electrode-Solution Interfaces. Annual Review of Physical Chemistry, 2021, 72 (1), pp.189-212. 10.1146/annurev-physchem090519-024042 . hal-03212310

\section{HAL Id: hal-03212310 https://hal.sorbonne-universite.fr/hal-03212310}

Submitted on 29 Apr 2021

HAL is a multi-disciplinary open access archive for the deposit and dissemination of scientific research documents, whether they are published or not. The documents may come from teaching and research institutions in France or abroad, or from public or private research centers.
L'archive ouverte pluridisciplinaire HAL, est destinée au dépôt et à la diffusion de documents scientifiques de niveau recherche, publiés ou non, émanant des établissements d'enseignement et de recherche français ou étrangers, des laboratoires publics ou privés. 


\author{
Molecular Simulation of Electrode-Solution Interfaces \\ Laura Scalfi, ${ }^{1}$ Mathieu Salanne, ${ }^{1,2}$ and Benjamin Rotenberg ${ }^{1,2}$ \\ ${ }^{1}$ Sorbonne Université, CNRS, Physicochimie des électrolytes et Nanosystèmes Interfaciaux, \\ F-75005 Paris, France; email: benjamin.rotenberg@sorbonne-universite.fr \\ ${ }^{2}$ Réseau sur le Stockage Electrochimique de l'Energie (RS2E), FR CNRS 3459, 80039 Amiens Cedex, France
}

(Dated: Aug. 2020)

\begin{abstract}
Many key industrial processes, from electricity production, conversion and storage to electrocatalysis or electrochemistry in general, rely on physical mechanisms occurring at the interface between a metallic electrode and an electrolyte solution, summarized by the concept of electric double layer, with the accumulation/depletion of electrons on the metal side and of ions on the liquid side. While electrostatic interactions play an essential role on the structure, thermodynamics, dynamics and reactivity of electrode-electrolyte interfaces, these properties also crucially depend on the nature of the ions and solvent, as well as that of the metal itself. Such interfaces pose many challenges for modeling, because they are a place where Quantum Chemistry meets Statistical Physics. In the present review, we explore the recent advances on the description and understanding of electrodeelectrolyte interfaces with classical molecular simulations, with a focus on planar interfaces and solvent-based liquids, from pure solvent to water-in-salt-electrolytes.
\end{abstract}

PACS numbers:

\title{
Contents
}

\section{INTRODUCTION}

II. HOW TO DESCRIBE ELECTRODE-SOLUTION INTERFACES IN MOLECULAR SIMULATIONS?

A. To be or not to be a metallic electrode

1. Insulating vs conducting

2. Electrodes in classical molecular simulations

3. Representing the electronic response of the metal

4. Focus on fluctuating charge methods

B. It takes two to tango

1. Description of the electrolyte

2. Electrode-electrolyte: non-electrostatic interactions

III. HOW TO SIMULATE POLARIZED ELECTRODE-SOLUTION INTERFACES

A. Increase tension (between electrodes) or handle (the electrolyte) with care

1. Simulation setup

2. Constant-voltage or finite field

B. Enough is enough? Sampling configurations

1. Electroneutrality

2. Statistical mechanics of the constant-potential ensemble

3. Importance sampling

IV. WHAT CAN WE LEARN FROM MOLECULAR SIMULATIONS OF ELECTRODE-SOLUTION INTERFACES?
A. Capacitance
B. Interfacial structure
C. Interfacial dynamics
D. Towards electrochemistry

VI. FUTURE ISSUES 


\section{INTRODUCTION}

Many key industrial processes, from electricity production, conversion and storage [1], to electrocatalysis or electrochemistry in general [2], rely on physical mechanisms occurring at the interface between a metallic solid (electrode), allowing the transport of electrons, and an electrolyte solution, in which electric currents may arise from the transport of ionic species or the orientation of polar molecules. The most interesting features of such electrode-solution interfaces emerge from the coupling between the charges accumulated on both sides, summarized by the concept of "Electric double layer" (EDL) [3]. From a classical perspective, in perfect metals the electronic charge is localized at the surface and the electric potential is uniform inside the solid, so that the interfacial properties are essentially governed by the ionic densities and electrostatic potential profiles near the interface, which result from the balance between energetic considerations (attraction of counterions to the surface charge and repulsion of co-ions, favoring the build-up of charge) and entropic ones (diffusion leading to uniform concentrations). Of particular interest are of course the charge accumulated on the electrode and the capacitance of the interface, i.e. the derivative of the charge with respect to the potential drop across the interface, which can be measured in electrochemical experiments. Voltage also provides a handle on wetting properties, by changing surface free energies and the resulting contact angle.

While electrostatic interactions play an essential role on the structure, thermodynamics, dynamics and reactivity of electrode-electrolyte interfaces, these properties also crucially depend on the nature of the ions and solvent, as well as that of the metal itself. The finite size of the ions and of solvent molecules results in a layered structure near solid walls, and their ability to form hydrogen bonds (e.g. for water) constrains their orientation. Such features, which can be investigated using spectroscopic techniques, depend on the atomic lattice of the metal and a given fluid behaves differently on different faces of the same crystal of a given metal. Finally, many real materials cannot be considered as perfect metals, and the charge and potential distribution within the electrode and their coupling with the liquid must also be taken into account. This effect of the metallic character of the electrode on the properties of the interfacial liquid is a good illustration of the challenge that such interfaces pose for modeling, because by bridging electrons in a solid and ions in a solvent, they are a place where Quantum Chemistry meets Statistical Physics.

On the theoretical side, much progress has been made since the pioneering works of Gouy, Chapman and Stern. At the same continuous level of description, extensions of the mean-field Poisson-Boltzmann theory have been proposed to capture the effects of electrostatic correlations and excluded volume or solvent polarization [4, 5] on the structure and capacitance of the EDL, with a low computational cost compatible with routine use in engineering applications. Even the charging dynamics can be investigated at this level [6], even though the effects of ionic correlations or of the coupling with the solvent dynamics are more accurately described by mesoscopic simulations with explicit or implicit ions [7-10]. At the other extreme, quantum calculations, usually based on electronic Density Functional Theory (even though Quantum Monte Carlo can now provide even more accurate results e.g. on water-carbon interactions [11]), allow to capture the density of states of the metal as well as a detailed description of a few interfacial molecules $[12,13]$. However, their computational cost prevents a fully molecular description of the EDL, and resort to a simplified description of the solvent (polarizable continuum) is the rule rather than the exception. As a result, classical molecular simulations have emerged as a powerful compromise between an atomic description and a computational cost allowing a sufficient sampling of relevant electrolyte configurations.

In the present review, we explore the recent advances on the description and understanding of electrode-electrolyte interfaces with classical molecular simulations. While many applications involve porous electrodes with disordered structures and complex electrolytes such as room temperature ionic liquids, we restrict ourselves to the simpler yet practically relevant and physically rich case of planar interfaces and solvent-based liquids, from pure solvent to waterin-salt-electrolytes (we refer the readers to e.g. Refs. [14-18] on the porous and/or ionic liquid cases). In Section II, we discuss the description of electrode-solution interfaces, emphasizing the choice of the models to represent the metallic character of the electrode and its interactions with the electrolyte solution. Section III then presents the various strategies to simulate electrochemical systems, with a potential difference between two electrodes, and fundamental issues related to such simulations. Finally, Section IV illustrates a selection of properties which can be investigated with such classical molecular simulations, with examples on the capacitance, the interfacial structure and dynamics, as well as steps towards electrochemistry. 


\section{HOW TO DESCRIBE ELECTRODE-SOLUTION INTERFACES IN MOLECULAR SIMULATIONS?}

We begin with an overview of models used in classical molecular simulations of electrode-electrolyte interfaces, emphasizing first the description of the electrode (Section II A) and how to capture the electronic response of the metal at this level of description. We then turn in Section II B to the description of the electrolyte and the nonelectrostatic interactions with the electrode.

\section{A. To be or not to be a metallic electrode}

\section{Insulating vs conducting}

The question of how to model metallic electrodes in classical molecular simulations is related to the more general question of the electrostatic response of a medium to an electric charge (ion, or partial charges from molecules), encountered not only in electrochemistry, but in all systems involving interfaces, including e.g. biological macromolecules. From a quantum mechanical perspective, electric conduction in a medium is related to the position of the Fermi level relative to the system's energy levels: In a metal, the conduction band is thermally accessible to the electrons, which can be delocalized over the whole material, whereas in an insulator a band gap hinders the conduction of electrons, which remain localized on atomic sites. From a classical continuum perspective, an essential feature of these interfaces is a contrast in the polarization response of the various media, quantified by their dielectric constant $\epsilon_{r}$, ranging from 1 for vacuum to $\approx 80$ for liquid water and $\infty$ for a perfect metal. Such a dielectric contrast has strong consequences on the behavior of a charge distribution close to the interface. This is usually expressed in terms of image charges. For a sharp flat interface between two media 1 (polar solvent) and 2 (solid wall) with dielectric constants $\epsilon_{1}$ and $\epsilon_{2}$, the electrostatic potential arising from a set of charges (ions) $\mathbf{q}^{\text {ext }}=\left\{q_{1}^{\text {ext }}, \ldots, q_{N}^{\text {ext }}\right\}$ embedded in medium 1 is identical, within this medium, to that arising from a fictitious system in which medium 2 is assigned a dielectric constant $\epsilon_{1}$ and a set of image charges are placed symmetrically with respect to the boundary (see Figure 1a below), with magnitudes:

$$
\mathbf{q}^{i m}=\frac{\epsilon_{1}-\epsilon_{2}}{\epsilon_{1}+\epsilon_{2}} \mathbf{q}^{e x t}
$$

For an insulating interface such as water-vacuum $\left(\epsilon_{1} \gg \epsilon_{2}\right)$ the image charges are similar to the source charges $\mathbf{q}_{i m} \approx \mathbf{q}^{e x t}$, whereas for a perfect metallic interface $\left(\epsilon_{2} \rightarrow \infty\right)$ the images have opposite charges $\mathbf{q}_{i m}=-\mathbf{q}^{e x t}$. It follows a radically different electrostatic interaction of a charge with its image: attractive for the metallic case and repulsive for insulators.

\section{Electrodes in classical molecular simulations}

To model these systems at the atomic scale, one should in principle perform quantum calculations taking into account the electronic density on both the electrode material and the electrolyte. However, this becomes computationally prohibitive for the simulation of large systems over long time scales and alternative approaches have been developed to capture the effect of a metallic electrode on an electrolyte within classical simulations. The purpose of these models is not to provide an accurate description of the metal and its properties, but rather to reproduce the appropriate boundary conditions for the electrolyte. Within such a simplified description, it becomes possible to sample the configurations explored by the electrolyte at finite temperature and to investigate the properties of electrochemical interfaces. In this context, two features are particularly important: accounting for the polarization of the metal by the electrolyte, discussed in the following section, and the possibility to accumulate a net charge on the interface, e.g. in the presence of an applied voltage between two electrodes.

A first method to describe charged electrodes is to explicitly put a constant net charge on the electrode, using a surface charge on a wall [19-22] or discrete point charges [23, 24]. The electroneutrality of the system can be balanced by excess ions in the electrolyte or by an opposite charge on a second electrode. This setup would correspond to a charged pore or an isolated (open circuit) charged capacitor. From the electrostatic point of view, two oppositely and homogeneously charged walls induce a uniform electric field between them, so that it is (at least in principle) equivalent to directly apply an external electric field on the liquid confined in the electrochemical cell [25-29]. In a real system, however, the system is rather connected to a voltage generator, which maintains a constant electric potential difference between the electrodes and allows the exchange of charge between them [21, 22, 30-34]. We will discuss the corresponding simulation setups in Section III, and now turn to the representation of polarization of the metal in classical molecular simulations. 
Beyond their net charge, a characteristic feature of metallic electrodes is their polarization by the electrolyte (this is even the main effect for neutral electrodes). In the context of molecular simulations, one is primarily concerned with the effect of the metal on the electrolyte and a variety of methods have been proposed. The typical slab geometry of capacitors allows a number of simplifications, including analytical expressions of the electrostatic forces acting on the electrolyte charges and of the electrostatic energy. Even though a uniform charge distribution with varying magnitude can be used to account for an applied external field or voltage, such a description lacks the lateral charge heterogeneities induced by the discrete nature of ions and molecules of the electrolyte. From the electrostatic point of view, an efficient strategy is to impose the proper electrostatic boundary conditions at the surface of the metal using the above-mentioned concept of image charges, which can be either explicit or accounted for implicitly in modified Green functions [19, 28, 34-38]. The use of periodic boundary conditions (see section III) also requires special care to compute electrostatic interactions, and efficient algorithms have been developed to deal with the image charges in simulations [39]. Alternatively, the electrostatic problem can also be solved numerically without resorting to image charges: The Induced Charge Computation (ICC) method treats the charge density of the solid as a dynamical variable discretized on a grid and solves the Poisson equation to obtain the induced (surface) charge, as illustrated in Figure 1b. The dielectric medium is then characterized by a space-dependent dielectric constant $\epsilon(\mathbf{r})$ which can in principle describe arbitrarily shaped interfaces and non-homogeneous media. Based on the variational procedure of Allen et al. for solid-electrolyte interfaces [40], extensions using a matrix formulation [41] or an iterative algorithm $\left(\right.$ ICC $\left.^{*}\right)[42,43]$ were proposed. Another example of this numerical approach to induced charges is the Generalized Minimal Residue (GMRES) method, which provides good performance when used in conjuction with fast Ewald solver [44].

(a) Image charges

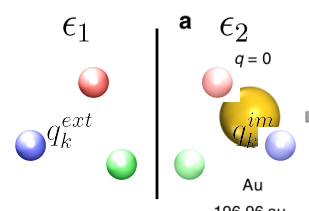

(b) Poisson solvers

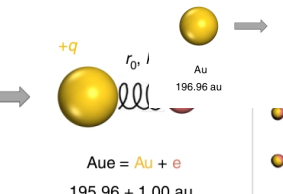

(c) Mobile charges

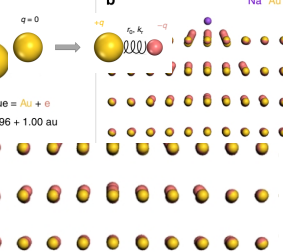

(d) Fluctuating charges

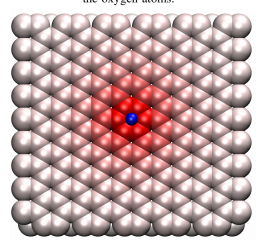

FIG. 1: Strategies to account for the polarization of the metal by the electrolyte in molecular simuations. (a) Image charges $q^{i m}$ inside the solid with permittivity $\epsilon_{2}$ induced by the electrolyte charges $q^{\text {ext }}$ in a medium of permittivity $\epsilon_{1}$ (see Eq. 1). (b) Solving the Poisson equation on a grid to compute the induced (surface) charges. (c) Describing the electronic response with mobile charges: here gold atoms are modeled using opposite charges tethered by a spring, with the cores representing the nuclei (yellow) fixed and the shells (pink) moving in response to the presence of a sodium ion (purple). (d) Describing the electronic response with fixed sites with fluctuating charges: here the charge of atoms in a graphite electrode is distributed inhomogeneously (darker red means more negative) in response to the presence of a cation such as $\mathrm{Li}^{+}$(blue). Panel b reproduced from Ref. [42], J. Chem. Phys. 2010, 132, 154112, with the permission of AIP Publishing; panel c reproduced from Ref. [45], Nature Commun. 2018, 9, 716, with permission of Springer Nature.

In order to include the molecular details of the interface (both in the shape and its atomistic nature), one can turn to descriptions based on the electrode atoms, i.e. treating the metal at the same level of description as the electrolyte, albeit with dedicated force fields. A first class of such descriptions consists in allowing mobile charges to rearrange in response to the configuration of the electrolyte: This includes core-shell models such as the Drude oscillator [45], with a charge tethered to the electrode atom via a spring (see Figure 1c), or the rod model [46, 47], in which the auxiliary charge can rotate at a fixed distance around the atom. Such models have the advantage of being easily implemented in standard molecular simulation codes. To ensure a correct adiabatic separation of the charge dynamics and avoid instabilities and/or energy transfers, the mass of the auxiliary charge and the spring strength or rod length should be carefully chosen. A second class of models, described in the following section, considers instead fixed but fluctuating charges, illustrated in Figure 1d. One advantage of all these atomistic descriptions compared to those based on image charges is that they are not restricted to slab geometries and can be used to deal with disordered porous electrodes. They capture the polarization effects by rearranging the charge distribution of the metal due to the electric potential created by the electrolyte at each step. While this is not completely in the scope of the present review, we finally briefly mention attempts at including simplified quantum mechanical treatments of the interface in classical simulations, e.g. based on the Jellium model, to represent the spilling of the electronic charge out of the electrode [48, 49], or the "direct dynamics" [50, 51]. Recent developments of mixed quantum/classical (QM/MM) simulations or tight binding approaches might also provide interesting alternatives in the near future. 


\section{Focus on fluctuating charge methods}

We now present in some more detail the family of models describing the polarization by assigning charge distributions (typically point charges or Gaussians) to the electrode atoms, with magnitude $\mathbf{q}=\left\{q_{1}, \ldots, q_{M}\right\}$ treated as additional degrees of freedom which fluctuate in response to the dynamics of ions and molecules of the electrolyte. Early models to account for the polarization of the metal by external charges in fact included both fluctuating charges and induced dipoles at the atomic sites as additional degrees of freedom [52, 53], but this idea doesn't seem to have been explored much further. The energy of the system is then expressed as a function of the positions $\mathbf{r}^{N}=\left\{\mathbf{r}_{1}, \ldots, \mathbf{r}_{N}\right\}$ and momenta of all electrolyte atoms (see also section IIB), and the charges $\mathbf{q}=\left\{q_{1}, \ldots, q_{M}\right\}$ are determined at each time step either by following an equation of motion, or by responding instantaneously to the motion of the electrolyte so as to impose the electric or electrochemical potential of the electrode atoms (see Section III).

This general idea has resulted in a variety of models, which to some extent share the same quadratic form of the energy as a function of the electrode charges q, even though the physical meaning of the parameters may differ, and of numerical algorithms to determine the charges at each time step. While fluctuating charge models had already been used for molecules, with the charge equilibration (QE) method or the electronegativity equalization method (EEM) [54-57], their use to represent a metallic surface was first described by Siepmann and Sprik [30], using Gaussian charge distributions on the electrode atoms. This model allowed them to simulate a water film near the tip of a scanning tunneling microscope and was then adapted within a Born-Oppenheimer framework to simulate an electrochemical cell by Reed et al. [31]. The electrostatic energy of the system reads

$$
\mathcal{U}_{e l}\left(\mathbf{r}^{N}, \mathbf{q}\right)=\frac{\mathbf{q}^{T} \mathbf{A q}}{2}-\mathbf{q}^{T} \mathbf{B}\left(\mathbf{r}^{N}\right)+C\left(\mathbf{r}^{N}\right)
$$

where the symmetric $M \times M$ matrix A depends on the positions of the electrode atoms and the parameters describing the charge distribution on each atom, while the components of the vector $\mathbf{B}$ are the electrostatic potentials due to the electrolyte on each electrode atom (see Refs $[31,58]$ for explicit expressions of $\mathbf{A}$ and $\mathbf{B}$ in the particular case of Gaussian charge distributions with 2D Ewald summation), and the scalar $C$ corresponds to electrostatic interactions within the electrolyte. The set of electrostatic potentials on each electrode atom is given by the gradient of $\mathcal{U}_{e l}$ with respect to $\mathbf{q}^{T}$

$$
\frac{\partial \mathcal{U}_{e l}\left(\mathbf{r}^{N}, \mathbf{q}\right)}{\partial \mathbf{q}^{T}}=\mathbf{A q}-\mathbf{B},
$$

and depends on the positions of the electrode atoms and on the electrolyte configuration. As a result, the set of charges satisfying the constraint of fixed electrostatic potentials $\Psi=\left\{\Psi_{1}, \ldots, \Psi_{M}\right\}$ for each atom, typically the same value for all atoms belonging to a given electrode and a difference $\Delta \Psi$ between the values for both electrodes, is given by $\mathbf{q}=\mathbf{A}^{-1}(\mathbf{B}+\mathbf{\Psi})$. In practice, if the matrix $\mathbf{A}$ can be inverted numerically (this has to be done only once if the electrode atoms do not move), the charges are computed at each step by a simple matrix-vector multiplication. Other methods are possible, such as finding the charges by minimizing $\mathcal{U}_{e l}-\mathbf{q}^{T} \boldsymbol{\Psi}$ numerically, e.g. with the conjugate gradient method, or treating the fixed potential as a holonomic constraint [59].

The expression of the energy is similar in the case of the QE/EEM method, which was used to investigate electrochemical interfaces [60-65], but the expressions of the matrix $\mathbf{A}$ and vector $\mathbf{B}$ involve terms such as electronegativities and chemical hardnesses, instead of the purely electrostatic picture described above. It is also related to the split charge equilibration approach, which includes bond-specific terms in the energy [66]. Finally, Pastewka et al. pushed the concept of using fluctuating charges as a proxy for a quantum description even further, by also parametrizing the band-structure energy, which appears in the tight-binding approximation with self-consistent charges to describe the terms beyond the electrostatic interactions between them, as a function of $\mathbf{q}$ [67]. This allowed a purely classical description of various carbon electrodes, taking into account to some extent their different band structures. The potential of such a promising strategy doesn't seem to have been much exploited so far.

\section{B. It takes two to tango}

\section{Description of the electrolyte}

On the other side of the interface, the electrolyte can be represented using various levels of sophistication. In molecular dynamics simulations, two main families of models are generally employed: all-atom models, with intramolecular interactions or rigid bonds, or coarse-grained models, offering a compromise between computational cost (due to the reduced number of interacting sites and the use of larger timesteps) and accuracy [68]. In the case of electrochemical 
interfaces, coarse-grained models generally include between 4 and 10 atoms per grains [69], resulting in a decrease of the number of interaction sites by one order of magnitude compared to all-atom models (note that an alternative consists in using "united-atom" models in which only the hydrogen atoms are merged with heteroatoms to which they are bonded).

In both families of models, the intermolecular interactions will then determine most of the liquid properties. Many analytical expressions are used in order to account for the short-range repulsion between the electronic clouds, the dispersion effects and the electrostatic interactions. Concerning electrostatics, all the models employ partial charges which are distributed among the interaction sites of the molecules, which interact between them and with the electrode charges. In a few cases, polarization effects are included by adding either charge-on-a-spring (Drude oscillator model) or induced dipoles on the atomic sites [70-72]. These simulations are more costly from the computational point of view since they involve additional degrees of freedom that have to be either propagated or determined by solving a set of self-consistent equations (they have a many-body character), but they should in principle be more accurate since the electrostatics interactions are of primary importance at the interface. An alternative is to use rescaled charges in order to mimic these polarization effects [73].

\section{Electrode-electrolyte: non-electrostatic interactions}

The electrostatic interactions between the electrode and the electrolyte ions and molecules follow from their respective descriptions as charge and dipole distributions, discussed above. Another crucial aspect is to account for the repulsive and dispersive intermolecular interactions between the electrolyte atoms and the electrode surface. In the earliest studies, the surface was materialized by a structure-less wall, that took the form of a hard wall, a purely repulsive potential or different flavors of one-dimensional Lennard-Jones potentials such as the Steele potential $[21,25,26,35]$. These descriptions are computationally less demanding but lack important molecular features close to the interface, that give rise $e . g$. to specific adsorption sites or templating effects. Atomic descriptions of the electrodes are therefore employed, using a finite number of discrete atoms, in order to account for different geometries and crystal structures [29, 30, 45, 47]. Usual intermolecular potentials are employed such as Lennard-Jones and Born-Mayer potentials. In most of these studies, the non-electrostatic electrode-electrode interactions are neglected, because the individual electrode atoms are immobile, with a few exceptions where the electrode structure is rigid but can translate as to mimic a piston at constant pressure, as done in Ref. [59]; in only a few cases, the electrodes atoms are free to move and interact with a harmonic potential [74]. It should be highlighted that the inclusion of explicit atoms for the intermolecular part of the interactions does not necessarily imply an atomistic treatment of the electrostatic part and vice versa. Mixing atomistic and structure-less descriptions however poses the problem of where to locate the interface (in a continuum picture) with respect to the atomic positions. Intermediate approaches have also been used, which formulate the interaction potential as a corrugated potential, avoiding the calculation of pair terms but reproducing the local roughness of the substrate [75].

As usual, the parametrization of these non-electrostatic interactions, which may lead or not to good predictions depending on how the electrostatic part is described, is crucial. While some force fields were fitted on ab-initio calculations or to reproduce experimental data, in the absence of reliable data the choices are often based on the availability of parameters from other studies. Accurate quantum Monte-Carlo reference data were reported for interfaces between water and graphene or carbon nanotubes [76], that could be used to develop new classical force fields for carbon electrodes, for which no specific optimization has been proposed to date. Comparatively, more efforts were put in the representation of metal surfaces. For example, Heinz et al. have proposed a systematic parameterization of LennardJones potentials for several face-centered cubic metals, that were shown to reproduce a few experimental data for interfaces with water, such as surface tensions [77]. When used in combination with a core-shell model to represent the polarization of the metal, it was necessary to reparametrize the Lennard-Jones potentials as well [45]. In the case of platinum, Siepmann and Sprik showed that using a three-body function was necessary to push water molecules on top of metal atoms to represent chemisorption effects [30]. An alternative approach was recently proposed to include these effects through an attractive two-body Gaussian potential [78]. We will also come back to reactive force fields to capture the breaking and formation of bonds in Section IV D.

\section{HOW TO SIMULATE POLARIZED ELECTRODE-SOLUTION INTERFACES}

Beyond the choice of the force field to describe the metallic electrode, the electrolyte and the interactions between them, a molecular simulation requires the definition of the simulated system and an algorithm to sample its configurations in order to compute physical properties. In this section, we explore more specifically the available options to simulate an electrochemical cell with an applied potential difference between two electrodes, as well as issues related 
to the sampling of the corresponding microscopic configurations. Even though the discussion below applies more generally, we illustrate the various points with a description of the electrode based on fluctuating charges. In addition, we will not discuss in detail the possibilities offered by existing open source softwares for the classical molecular simulation of electrochemical interfaces with the methods described in the present review, but refer the reader to the corresponding descriptions of e.g. Metalwalls [79], which is dedicated to the simulation of such interfaces, or more generic simulation packages allowing such simulations (possibly with an open source modification not provided in the standard distribution) such as LAMMPS, OpenMM or ESPRESSO (see [80] for links).

\section{A. Increase tension (between electrodes) or handle (the electrolyte) with care}

\section{Simulation setup}

In a typical electrochemical system, two electrodes are separated by a slab of liquid electrolyte with all dimensions along the surfaces and between them much larger than molecular ones. In order to ensure that the two interfaces do not interfere (even though the total charge accumulated on both electrodes are of course correlated, see also Section III B 1), the distance between the electrodes must be large compared to the screening length. In experiments, one also introduces a separator to prevent contact (hence short-circuit) between the electrodes. The typical dimensions of systems that can be simulated with classical molecular simulations is in the range of a few to a few tens of nanometers. This requires a simplified description of the real device and limits the range of physical systems that can be simulated in a meaningful way. In particular, one always neglects the presence of the separator, which is a safe assumption provided that the distance between the electrodes remains large compared to the screening length. This in turn sets a lower bound on the ionic concentrations that can be considered - typically $\sim 0.1$ mol $\mathrm{L}^{-1}$, a limit which also emerges from the constraint of having enough ions in the simulation box to ensure a good sampling of the phase space.
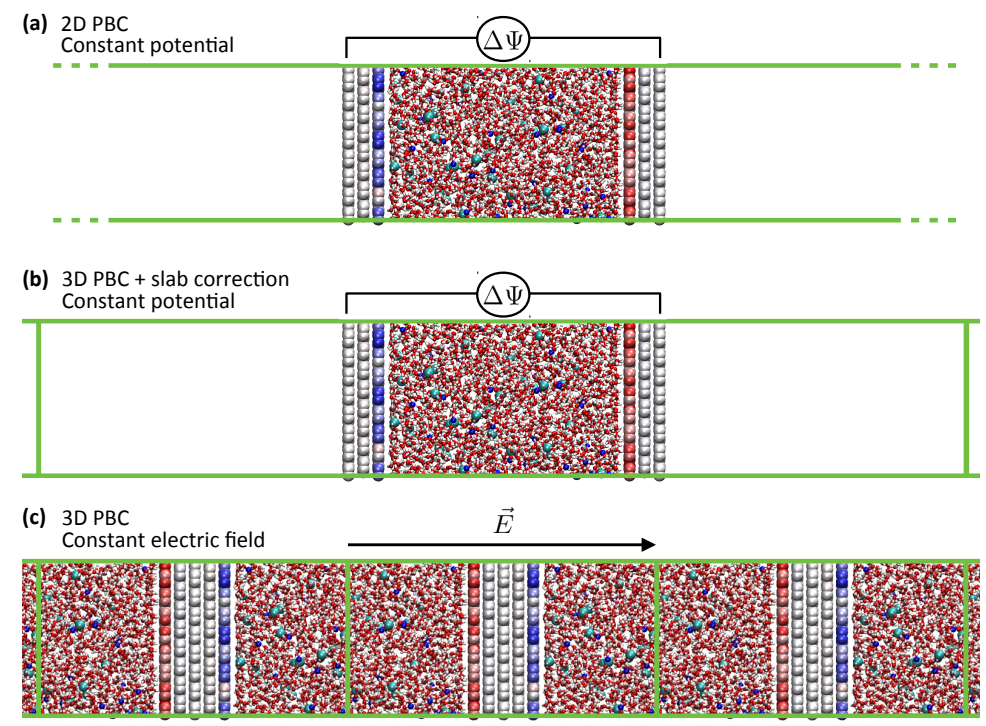

FIG. 2: Simulation setups to simulate a capacitor consisting of graphite electrodes separated by a 1 mol $\mathrm{L}^{-1}$ aqueous $\mathrm{NaCl}$ solution under a voltage $\Delta \Psi=2 \mathrm{~V}$; the color of the electrode atoms reflects their instantaneous charge. (a) Periodic boundary conditions (PBC) are applied only in the two directions along the surfaces, but not in the direction perpendicular to the electrodes, and the two electrodes are maintained at different potentials, corresponding to a voltage $\Delta \Psi$. (b) PBC are applied in all directions, but the simulation box also includes vacuum (here the length of the box in this direction is three times larger than the electrochemical cell) and a "slab" correction is applied when computing electrostatic interactions; as in the previous case, the two electrodes are maintained at different potentials. (c) PBC are applied in all directions, without vacuum; all electrode atoms are maintained at the same potential and the capacitor is charged by applying an electric field to the electrolyte such that $\Delta \Psi=-E L_{z}$, with $L_{z}$ the box dimension in the direction perpendicular to the electrode.

Last but not least, the small number of atoms in the system $\left(10^{3}-10^{5}\right)$ compared to a real device requires, as always in molecular simulation of condensed matter, the use of periodic boundary conditions (PBC). Based on the above discussion, it is natural to consider $\mathrm{PBC}$ in the two directions along the surfaces, but not in the direction perpendicular 
to the electrodes, as illustrated in Figure 2a. This requires in particular computing long-range electrostatic interactions with dedicated approaches, such as 2D Ewald summation, also taking into account the description of electrode atoms by Gaussian charge distributions instead of point charges if needed [31, 58]. An alternative approach, more easily implemented in standard packages, is to mimick 2D PBC by considering PBC in all directions, but adding vacuum to the simulation box, as illustrated in Figure 2b. In that case, it is necessary to apply a "slab" correction when computing electrostatic interactions [81].

\section{Constant-voltage or finite field}

The simulation of an electrochemical cell also requires a way to impose voltage between the two electrodes. The most straightforward way to achieve this, with descriptions allowing to set the potential of electrode atoms such as methods based on fluctuating charges, is to impose different values of the potential for atoms belonging to the two electrodes, i.e. one value for each electrode with a difference $\Delta \Psi$ between the two electrodes, as illustrated in panels 2 a and $2 \mathrm{~b}$ for the $2 \mathrm{D}$ and 3D-slab PBC cases, respectively. Recently, an alternative approach has been proposed to allow the use of 3D PBC without the need to introduce vacuum, hence decrease the computational cost, and facilitate the implementation in standard simulation packages. The method introduced by Dufils et al. [82], illustrated in Figure 2c, consists in applying a finite electric field to the electrolyte via an extended Hamiltonian. Such finite field methods, developed in the framework of the modern theory of polarization [83], had first been adapted to investigate EDLs near charged or polar insulator-electrolyte interfaces $[84,85]$. By imposing a constant potential on all the electrode atoms (there is a single electrode in this setup) using the fluctuating charge method and an electric field on the electrolyte such that $\Delta \Psi=-E L_{z}$, with $L_{z}$ the box dimension in the direction perpendicular to the electrode, one can charge the capacitor by creating two EDLs, one on each side of the electrode. If the width of the electrode is sufficient, one recovers the same results (in particular for the induced charges on the electrode or the structure of the interfacial electrolyte) as in the 2D periodic case of Figure 2a, with a reduced computational cost. A further benefit of this finite field approach is that it could easily be used with other methods to describe the metal (it had already been used in 3D PBC with the ICC method [39]) with explicit electrode atoms, e.g. with the core-shell model of Ref. [45], which capture the polarization of the metal but does not provide a handle to control the potential of the electrodes (hence the voltage between them), or even in ab initio simulations [86].

\section{B. Enough is enough? Sampling configurations}

While the previous section presented the difficulties related to the description of the system, we now turn to the theoretical and numerical challenges pertaining to the sampling of microscopic configurations of electrode-electrolyte interfaces. Molecular simulations are inherently related to Statistical Mechanics and the fundamental problem of sampling configurations from a thermodynamic ensemble, from which physical properties can be computed as averages. Even though most molecular simulations with constant-potential electrodes are performed in the canonical ensemble (constant number of electrolyte ions and molecules, $N$, volume $V$ and temperature $T$ ), one can in principle also consider a constant normal pressure on the electrodes (at least with rigid electrodes acting as pistons), or even grandcanonical (fixed chemical potential) and Gibbs ensemble (exchange between two systems) simulations. In the latter cases, which involve the exchange of particles, it might be necessary to resort to Monte Carlo simulations, as done e.g. in Refs. [21, 22, 87, 88] instead of molecular dynamics more commonly employed in this field. We focus here on three separate though related aspects, which apply generally to all the above situations, but limiting ourselves to the more common canonical ensemble: the issue of global electroneutrality, the statistical mechanics of the constant-potential ensemble, and importance sampling.

\section{Electroneutrality}

When studying an electrochemical system with electrodes held at a constant potential difference by a generator at a classical molecular dynamics level, the main focus and interest is rather on the electrolyte properties. Importantly, the details of the device that applies a potential difference are not taken into account. Instead the models consider an open system, very much like in a grand-canonical ensemble, exchanging charge (instead of particles) with a reservoir. In order to properly separate the electrochemical cell from the electric potential generator, the interaction between them should be negligible. This requires both subsystems to be electroneutral so as to cancel the leading term in the long-range electrostatic interaction. Although in the real device there are fluctuations and the electroneutrality of the cell is only verified on average, it should be enforced at a microscopic level in molecular simulations not explicitly 
including the charge reservoir. This can be achieved easily in descriptions with fluctuating charges by adding a Lagrange multiplier to enforce this constraint when computing the charges of the electrode atom. This multiplier corresponds to a potential shift applied to both electrodes simultaneously, while keeping the potential difference unchanged $[59,89,90]$.

\section{Statistical mechanics of the constant-potential ensemble}

The natural ensemble associated with the above-mentioned simulations is the canonical constant-potential ensemble, characterized by the total number of atoms $N$, the volume $V$, the temperature $T$ and the applied potential $\boldsymbol{\Psi}$ (more precisely, the set of potentials imposed no each electrode atom, usually one value per electrode). The charge distribution on the electrode atoms is then the conjugate variable of the applied potential and its fluctuations contain useful information. The aim of molecular simulations is to sample configurations from this ensemble in order to compute meaningful statistical averages. Along with approximations in the choice of force fields and the level of description of the quantum degrees of freedom, there are approximations related to how the chosen algorithm samples this thermodynamic ensemble. Using constant charges on electrode atoms ignores any charge fluctuation around the mean. One can use external inputs or post-processing to link the total electrode charge to an equivalent applied potential, but the configurations are not sampled according to their weight in the constant-potential ensemble.

Even though in principle one could also use Monte Carlo algorithms to sample the electrolyte configurations and electrode charges, to date most simulations using fluctuating charge models used molecular dynamics. In addition, except in earlier studies using Car-Parrinello (CP) dynamics with a fictitious mass for the additional degrees of freedom [30], the vast majority of simulations employing fluctuating charge models rely instead on Born-Oppenheimer (BO) dynamics, by assuming a separation of time scales between the electronic and nuclear degrees of freedom [31]. This allows using larger time steps, but reduces the full phase space to that of electrolyte configurations, since to any such configuration corresponds a single set of electrode atom charges $\mathbf{q}^{*}$. A thorough discussion of the statistical mechanics of the constant-potential ensemble can be found in Ref. [90], where we clarified in particular which observables can be computed exactly using BO dynamics on the charges. This is e.g. the case of the average total charge of an electrode $\left\langle Q_{t o t}\right\rangle=\left\langle Q_{t o t}^{*}\right\rangle$. In contrast, the variance of the total charge, $\left\langle\delta Q_{\text {tot }}^{2}\right\rangle$, which is related to the differential capacitance by [21, 91]:

$$
C_{\text {diff }}=\frac{\partial\left\langle Q_{t o t}\right\rangle}{\partial \Delta \Psi}=\beta\left\langle\delta Q_{\text {tot }}^{2}\right\rangle
$$

with $\Delta \Psi$ the voltage between the two electrodes and $1 / \beta=k_{B} T$ the thermal energy, includes a contribution from the suppressed thermal charge fluctuations. The differential capacitance is then given by

$$
C_{\text {diff }}=C_{\text {diff }}^{\text {electrolyte }}+C_{\text {diff }}^{\text {empty }}=\beta\left\langle\delta Q_{\text {tot }}^{* 2}\right\rangle+C_{\text {diff }}^{\text {empty }}
$$

where $\left\langle\delta Q_{t o t}^{* 2}\right\rangle$ is the variance sampled within BO dynamics and $C_{\mathrm{diff}}^{\text {empty }}$, for which an explicit expression can be found in Ref. [90], corresponds to the empty capacitor (i.e. in the absence of thermal fluctuations of the electrolyte).

\section{Importance sampling}

As usual in molecular simulations, one may also face sampling issues due to physical processes that result in long time scales not accessible by straightforward simulations. This includes $e . g$. slow transport within the electrolyte, or adsorption/desorption at the interface, or even phase transitions. In that case, one can resort to dedicated approaches, such as umbrella sampling to compute free energies, or transition path sampling to compute rates and analyze mechanisms. Examples of studies in the context of electrolyte interfaces, illustrated in Section IV C, involve collective variables such as the distance of an ion to the surface or between two ions to investigate their adsorption/desorption and pair formation/dissociation, or the number of water molecules in a probe volume, to investigate the hydrophilic/phobic behavior of the interface.

An importance sampling approach more specific to electrode-electrolyte interfaces was proposed in Ref. [91]. By adapting the standard histogram reweighting approach to the case of constant-potential simulations, it is possible to combine simulations performed at different potentials in order to optimally compute the properties of the system as a function of voltage, including for voltages for which no simulations are performed. The only requirement is an overlap of the distributions of the total charge $P\left(Q_{t o t}\right)$, or the joint distribution $P\left(Q_{t o t}, p\right)$, with $p$ a property of interest such as density profiles or orientational distributions, between the simulations at various voltages. This approach has been used for example in the study illustrated in Section IV B. Even though to the best of our knowledge it has 
never been done to date, one could use the same idea to derive a parallel tempering algorithm, exchanging replicas between simulations at different potentials (instead of temperature in the original method) to enhance the sampling of phase space. Histogram reweighting has also been used recently in Ref. [92] to sample the so-called vertical energy gap related to electron transfer reactions (see Section IV D). Other strategies not requiring an explicit biais on the collective variable of interest, but taking advantage of statistical tools, include indirect umbrella sampling to reweight configurations using a bias on an auxiliary collective variable, which was applied in Ref. [93] to the Madelung potential experienced by ions at an electrochemical interface (see Section IV C).

\section{WHAT CAN WE LEARN FROM MOLECULAR SIMULATIONS OF ELECTRODE-SOLUTION INTERFACES?}

Once a description of the system and a sampling strategy are chosen, one can perform classical molecular simulations to compute observable properties. In this section, we provide a selection (hence by no means exhaustive list) of illustrations of such properties typical of electrode-electrolyte interfaces: capacitance, interfacial structure and dynamics and an opening towards electrochemistry. We also present in Supplemental Material an application to electrowetting. All examples have in common the use of a fluctuating charge model to describe the electrode.

\section{A. Capacitance}

We begin by considering one of the most relevant property for practical applications, namely the capacitance. More precisely, one generally applies a voltage $\Delta \Psi$ between two metallic electrodes separated by an electrolyte, and the charge $\pm Q_{t o t}$ on both electrodes, which fluctuates in response to the thermal motion of the liquid (see Section III B 2), allows the definition of an integral capacitance $C_{\text {int }}=\left\langle Q_{t o t}\right\rangle / \Delta \Psi$ and a differential capacitance $C_{\text {diff }}=\partial\left\langle Q_{t o t}\right\rangle / \partial \Delta \Psi$. Both quantities coincide only when the differential capacitance does not depend on voltage, i.e. when the response to applied voltage is linear. Such a linear behavior is expected from continuum electrostatics for polar liquids behaving as pure dielectric media and for dilute electrolyte solutions. However, the organization of the molecular solvent and ions at the interface do not exactly follow the assumptions leading to simple analytical expressions, and molecular simulations allow to evaluate $C_{\text {int }}$ and $C_{\text {diff }}$, as well as to correlate their evolution as a function voltage with structural changes in the interfacial fluid.

In practice, one could compute the average charge $\left\langle Q_{t o t}\right\rangle$ as a function of voltage and perform a numerical derivative to obtain the differential capacitance. A much more efficient and accurate approach is to use the fluctuation-dissipation relation Eq. 5, which provides $C_{\text {diff }}$ from the variance of the charge distribution in a single simulation. We show in the Supplemental Material an illustration of this method with results from Scalfi et al. for two capacitors consisting of graphite electrodes separated by pure water or a $1 \mathrm{M}$ aqueous $\mathrm{NaCl}$ solution [90]. For pure water, the abovementioned continuum prediction for the capacitance, taking the permittivity of the SPC/E water model used in this work, results in a value 3-4 times larger than the one obtained from the simulations, because it fails to account for the efficient screening of the field by the first layers of interfacial molecules [94-96]. Adding salt increases the capacitance compared to pure water, but this increase is moderate for the considered concentration and inter-electrode distance. As for the pure water case, the above-mentioned continuum prediction is not accurate, as expected for Debye-Hückel theory at such a high concentration. The shortcomings of such continuous descriptions to estimate the capacitance (both for pure solvent and electrolyte solutions) reflect their limitations to account for the detailed organization of the interfacial fluid, which can also be investigated by molecular simulation, as discussed in the next section.

\section{B. Interfacial structure}

One of the most straightforward yet valuable information provided by molecular simulation is the microscopic structure of the system. In the case of planar electrode-electrolyte interfaces, density profiles as a function of the position $z$ in the direction normal to the interface can be computed from the trajectories as an ensemble average as $\rho_{\alpha}(z)=\left\langle\sum_{i \in \alpha} \delta\left(z_{i}-z\right)\right\rangle$, with $\delta$ the Dirac delta function and where the sum runs over atoms $i$ of type $\alpha$. Similar definitions can be used to compute (1D, 2D or 3D) number, charge or polarization density profiles, distributions of molecular orientation, radial distribution functions, coordination numbers, etc. From the charge density profiles, one can also obtain electrostatic potential profiles by integrating the 1D Poisson equation, to make the link with the interfacial capacitance (in the presence of ions, using the potential drop across each interface) or the dielectric permittivity of the liquid (in the absence of ions, using the slope of the potential in the bulk region). The insights from molecular simulations are particularly valuable, since they capture explicitly the effects of the discrete nature 
of ions and solvent molecules, which lead in particular to their layering near the planar electrode, and of their complex interactions (steric, electrostatic, dispersion, ...). This allows a detailed investigation of the composition and organization of the interfacial liquids and their evolution as a function of applied voltage. The results can also serve as reference data for simpler theories, such as the ones mentioned in the introduction.
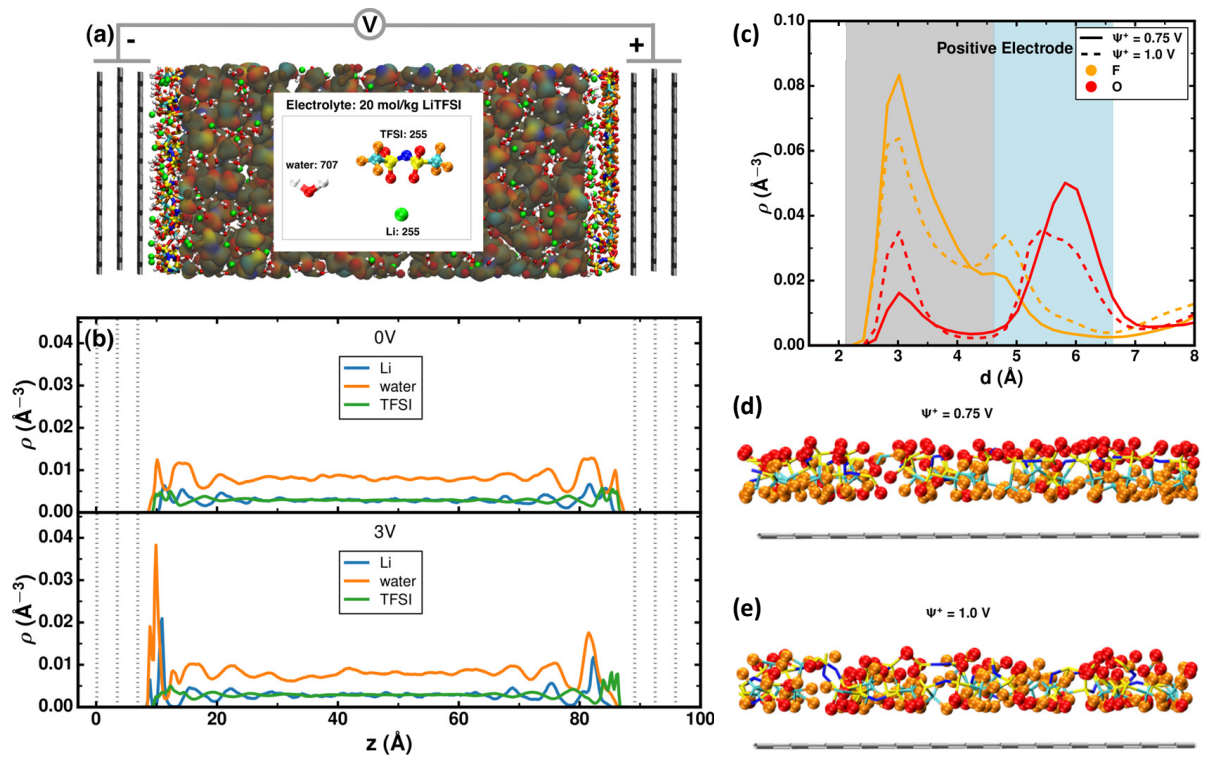

FIG. 3: (a) Capacitor consisting of graphite electrodes separated by a water-in-salt-electrolyte (20 mol/kg aqueous LiTFSI solution). An all-atom model is used, with $\mathrm{H}$ (white), O (red), Li (green), F (orange), C (cyan), S (yellow), and N (blue) atoms, but for clarity the TFSI anions beyond interfaces are represented by isosurfaces. (b) Density profiles for Li (blue), water (orange) and TFSI (green) at $\Delta \Psi=0 \mathrm{~V}$ (top) and $3 \mathrm{~V}$ (bottom). (c) Density profiles for $\mathrm{O}$ and $\mathrm{F}$ from TFSI anions on the positive electrode, for $\Delta \Psi=1.5$ and $2 \mathrm{~V}$; the shaded areas indicate the first and second adsorbed layers; the key indicates the potential of the positive electrode, assumed to be $\Psi^{+}=\Delta \Psi / 2$ ). (d,e) Snapshots illustrating the structure close to the positive electrode from simulations at $\Delta \Psi=1.5$ and 2 V. Adapted with permission from Ref. [97], J. Phys. Chem. C 2018, 122, 23917, Copyright (2018) American Chemical Society.

As an illustration, we consider here the complex case of a water-in-salt electrolyte (WiSE), proposed recently as promising for high-voltage batteries and supercapacitors [98, 99], by reporting some results of Li et al. [97]. Figure 3a shows a system consisting of a $20 \mathrm{~mol} / \mathrm{kg}$ aqueous LiTFSI solution between two graphite electrodes maintained at a constant potential using the method described in section II A 4. In such an electrolyte, the number of water molecules per ion pair is lower than 3 (compared to $\approx 50$ for a typical $1 \mathrm{~mol} / \mathrm{L}$ concentration), so that standard EDL theories are not expected to hold. The density profiles in Figure $3 \mathrm{~b}$ clearly illustrate the layering of the interfacial fluid even in the absence of applied voltage and a bulk region far from the surfaces, as well as the change in composition and structure of both interfaces under voltage. One can note in particular the increase in water concentration near the negative electrode (left) accompanying that of $\mathrm{Li}^{+}$, which approach the surface with their limited hydration shell. The structure on the positive electrode is further illustrated in panel 3c, which shows the density profiles for oxygen and fluorine atoms from the TFSI anion for two voltages. The most dramatic structural change occurring at this surface between $\Delta \Psi=1.5$ and $2 \mathrm{~V}$ is the reorientation of the anions, leading to a closer approach of $\mathrm{O}$ atoms (carrying a larger partial charge than F) to the surface, also visible on the typical snapshots of panels 3d and 3e. Even though we do not discuss this further here, it was also found in this work, using importance sampling techniques, that changes in the interfacial composition and structure as a function of voltages could be linked to peaks in the differential capacitance [97], as previously reported for room temperature ionic liquids [16, 100] and concentrated electrolytes [101]. One can finally note that, even in such complex cases, molecular simulation results can be used as reference data to validate and parameterize simpler theories, as was done e.g. by McEldrew et al. in a contemporary study of a similar WiSE between uniformly charged walls [5]. 


\section{Interfacial dynamics}

Applications of electrode-electrolyte interfaces to energy storage (batteries, supercapacitors) not only aim at improving the amount of energy that can be stored, by increasing the capacitance of the interface or the voltage, but also at reducing the charge/discharge time to increase the power that can be delivered. In the standard $R C$ circuit picture, the charging time depends on the resistance of the bulk electrolyte and the interfacial capacitance, but not on the interfacial dynamics. The situation in realistic porous electrode materials is more complex and molecular simulation has contributed to a better understanding of the charging dynamics in these systems, also making the link with more involved equivalent circuit models used in electrochemistry [102-105]. However, even in the simpler case of a planar electrode and a solvent-based electrolyte, which is the focus of the present work, the dynamics of the interfacial fluid is modified by the presence of the solid. This in turn may modify not only the charging dynamics but also the kinetics of electron transfer reactions (which will be discussed in more detail in section IV D). We illustrate here some important findings on the dynamics at a water-platinum interface uncovered using molecular simulation.

(a)

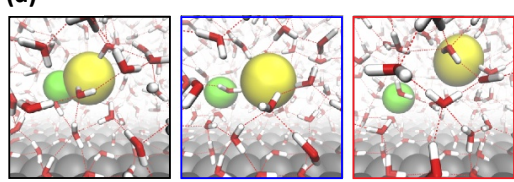

(b)

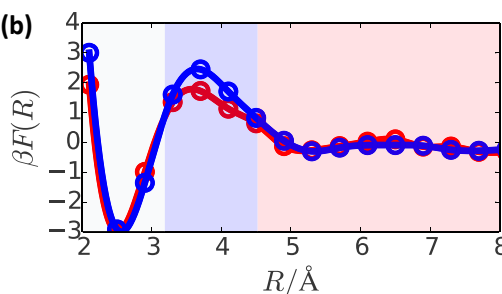

(c)

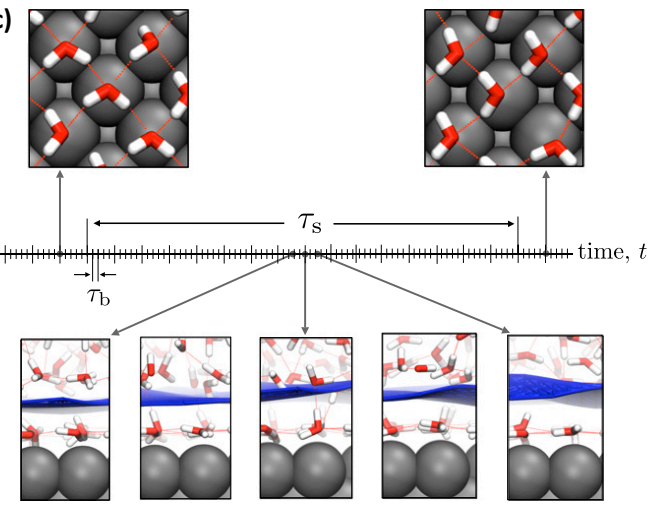

FIG. 4: (a) Typical configurations for the dissociation of an ion pair $\left(\mathrm{Na}^{+}\right.$in green, $\mathrm{I}^{-}$in yellow) in water near a (111) $\mathrm{Pt}$ electrode surface, from a contact ion pair (black-bordered panel) to a dissociated state (red-bordered panel). (b) Free energy profile as a function (in units of the thermal energy $k_{B} T=1 / \beta$ ) of the distance $R$ between the ions in the bulk (red) and near the surface (blue). (c) Illustration of the slow time scale $\tau_{s}$ associated with structural rearrangements of water near a (100) Pt surface, compared to that $\tau_{b}$ for the relaxation of bulk density fluctuations (small ticks are separated by 20 ps, larger than typical values for $\tau_{b}$ ). The blue surface in the bottom panels indicates the instantaneous interface between the first adsorbed layer and the rest of the fluid, which fluctuates due to local changes in the hydrogen bond pattern. Collective fluctuations eventually leading to global rearrangements such as between the top panels occur on much longer time scales. Panels (a) and (b) adapted with permission from Ref. [93], PNAS, 2017, 114, 13374; panel (c) adapted with permission from Ref. [106], with permission of $P N A S, 2013, \mathbf{1 1 0}, 4200$.

Kattirtzi et al. investigated the dissociation of ion pairs near a Pt electrode using classical (for $\left.\mathrm{Na}^{+}-\mathrm{I}^{-}\right)$and $a b$ initio (for water ions $\mathrm{H}_{3} \mathrm{O}^{+}-\mathrm{HO}^{-}$) molecular dynamics [93]. Figure 4a shows configurations for the $\mathrm{Na}^{+}-\mathrm{I}^{-}$case near a (111) Pt electrode surface, modeled using the method described in section II A 4 and the force field of Siepmann and Sprik [30] for the water-Pt interaction, which includes two and three body terms. The three configurations are typical for a contact ion pair, an intermediate state and a dissociated state, corresponding to the regions visible in the free energy profiles as a function of the interionic distance $R$, obtained by umbrella sampling (see section III B 3 ) and shown in panel $4 \mathrm{~b}$. This panel further shows that the free energy profile along this distance $R$ is in fact only slightly affected by the vicinity of the surface, with no change in the relative free energies of the states (suggesting limited changes in their solvation structure with respect to the bulk) and a small increase in the activation barrier for the dissociation/recombination, which would suggest only a slight decrease in the corresponding rates. However, as in the bulk [107], the interionic distance is not sufficient to examine the mechanism. It also involves collective water fluctuations, which were described by Kattirtzi et al. using a simple collective variable, namely the Madelung potential on the ions. From the change in free energy barrier including this variable (with an increase of $1.5 k_{B} T$ with respect to the bulk), one would predict a decrease in the dissociation rate near the electrode $k_{\text {elec }} \approx 0.2 k_{b u l k}$. However the dissociation rate measured in the simulations is in fact $k_{\text {elec }} \approx 0.02 k_{b u l k}$, i.e. the decrease is ten times larger. This is because the free energy is not sufficient to predict the rate, as the dynamics of water fluctuations also plays a role [93].

The dynamics of water near a Pt surface was in fact considered in earlier work by some of the same authors [106, 108110]. It was found that these dynamics can be highly collective, heterogeneous and slow. Figure 4c illustrates the slow time scales associated with structural rearrangements of water near a (100) Pt surface, compared to that for the 
relaxation of bulk density fluctuations. On this particular surface, water molecules in the first adsorbed layer form an ordered network of strong H-bonds between them. The lack of interactions with molecules beyond this layer (see the bottom parts of this panel) in fact results in an unexpected hydrophobic behavior, with e.g. a large contact angle of a water droplet on this adlayer. From the dynamical point of view, switching between distinct but equally probable H-bond patterns (see top parts of Figure 4c) requires collective rearrangements, associated with very long time scales and displaying dynamical heterogeneity [106]. While some results observed on this particular surface should apply generally to other Pt surfaces and other metals, the details of the dynamics crucially depend on the interplay between the geometry of the solid lattice and the interactions with the fluid, so that molecular simulation is ideally suited for such investigations. It was for example used recently to analyze the reorientation dynamics of water molecules at an electrified graphene interface [111].

\section{Towards electrochemistry}

In general, simulating reactive systems is challenging in molecular simulation: Reactive force fields need to be employed, which are difficult to parameterize. Nevertheless, many electrochemical reactions do not involve bond breaking/formation, but only a change of the redox state of the species. This observation is central in Marcus theory, which was developed in order to calculate reaction free energies for electron transfer [112]. In short, this classical transition state theory introduces a key quantity, the solvent reorganization energy, associated with the solvent rearrangement when the redox state of the solute changes. In classical molecular simulation, Warshel introduced the vertical energy gap, that is the potential energy difference between the oxidized and the reduced species at fixed solvent coordinates, as the key reaction coordinate for simulating electrochemical reactions [113].

Although this development allowed straightforward studies of electron transfer reactions in bulk solutions, in an electrochemical cell setup an additional complexity arises because the electrons are transferred to the electrodes instead of vacuum. Madden and co-workers proposed an approach in which the constant applied potential condition is enforced before and after the electron transfer reactions, leading to different charge organizations inside the electrodes [114]. Using this approach, they showed how the solvent reorganization energy, and hence the free energy curves, depend on the applied potential and on the distance of the redox species from the electrode in a molten salt. Surprisingly, the variations they observed where much smoother than expected from the pronounced oscillations in the mean electrical potential inside the double-layer region, a result which was confirmed in further studies [115].

The next challenge for classical simulations relies on the study of active interface. First steps in this direction were made using the Empirical Valence Bond method to describe the transfer of proton in water on silver or platinum electrodes [116, 117]. Switching in electrometallization cells has also been studied using reactive force fields (REAXFF) together with the charge equilibration method for the electrostatic part [61,62]. The electrodes, made of copper, were allowed to dissolve inside an amorphous silica and redeposit upon application/cancellation of a voltage, eventually leading to the formation of a conductive contact between the two electrodes. The extension of this approach to more conventional solvents is yet hindered by the difficulty of developing accurate reactive force fields for complex systems. Another strategy was recently proposed, in which the charge transfer is modeled as a stochastic process, using methods borrowed from grand canonical Monte Carlo simulations [118]. We can therefore expect that more simulations of reactive electrochemical interfaces will be proposed in future years.

\section{SUMMARY POINTS}

1. Over the last 30 years, classical molecular simulations have emerged as an essential tool to investigate the properties of electrode-electrolyte interfaces: By providing a compromise between an atomic description and a computational cost allowing a sufficient sampling of relevant electrolyte configurations, they offer the possibility to face the challenge posed by such interfaces bridging electrons in a solid and ions in a solvent, i.e. Quantum Chemistry and Statistical Physics.

2. We have provided an overview of the models and methods to describe the metallic character of the electrode and its interactions with the electrolyte solution, and we discussed simulation setups, algorithms and statistical tools to sample configurations from the statistical ensemble corresponding to constant-potential electrodes.

3. We have illustrated a selection of properties which can be investigated with classical molecular simulations, with examples on the capacitance, the interfacial structure and dynamics, electrowetting, as well as steps towards electrochemistry. 
4. The results obtained at this classical level can be compared directly to experiments probing the interface on the molecular scale, but also serve as reference data for simpler theories of electric double layers, generally used to interpret macroscopic electrochemical experiments.

5. Even though we restricted ourselves to the simpler yet practically relevant and physically rich case of planar interfaces and solvent-based liquids, from pure solvent to water-in-salt-electrolytes, some of the methods discussed here also apply to more complex (e.g. disordered nanoporous) electrodes and to solvent-free electrolytes (room temperature ionic liquids).

\section{FUTURE ISSUES}

1. The classical description of a metallic electrode remains a challenging issue. For example, most electrode materials do not behave as perfect metals. Electrostatic screening inside the solid can be captured at the continuum level using Thomas-Fermi theory, an approach which can also be exploited for classical molecular simulation. Earlier attempts included this effect of screening on the electrolyte via an external potential [119, 120], but new strategies have been proposed very recently, based on fluctuating charges [121] or on mobile charges [122]. This will be particularly useful to address the effect of the metallic character of the electrode on the properties of the interfacial fluids, such as the nanoscale capillary freezing of ionic liquids [123].

2. The treatment of non-electrostatic interactions also offers opportunities for improvement. The flexibility of the electrode has almost never been addressed, even though it might play a role in particular in porous electrodes. Due to the importance of electrochemical reactions, it is likely that reactive force fields will also gain importance, if their parametrization can be extended to more complex systems. The availability of accurate reference data from $a b$ initio calculations, in particular quantum Monte Carlo, will allow a better parametrization of force fields based on existing models. One can also anticipate that the rapid development of Machine-Learning based approaches (e.g. force fields based on neural networks) in molecular simulation will also reach the community working on electrode-electrolyte interfaces.

3. Another promising strategy is offered by hybrid approaches, coupling several levels of descriptions. As an example, a mesoscopic description of the solvent, based on classical density functional theory, was recently coupled to a fluctuating charge model of a graphene electrode [96] and used to investigate electron transfer reactions in a classical solute [124]. In the other direction, the development of classical models of the interface might also help improving the coupling between the electrolyte and the electrode in QM/MM simulations.

4. From the physical point of view, all these developments should allow to investigate new phenomena such as the electro-mechanical couplings related to electrotunable lubricity [125] or Electrochemical Quartz Microbalance Experiments. The ability to take bond formation an breaking into account would also open the way to the study of the formation of the so-called solid electrolyte interphase (SEI), which plays a crucial role in batteries.

\section{DISCLOSURE STATEMENT}

The authors are not aware of any affiliations, memberships, funding, or financial holdings that might be perceived as affecting the objectivity of this review.

\section{ACKNOWLEDGMENTS}

The authors are grateful to many colleagues with whom they have worked or exchanged on this topic over the years, in particular Paul Madden, Michiel Sprik, David Limmer, Céline Merlet. This project has received funding from the European Research Council (ERC) under the European Union's Horizon 2020 research and innovation programme (grant agreement No. 771294 and grant agreement No. 863473). This work was supported by the French National Research Agency (Labex STORE-EX, Grant ANR-10-LABX-0076, and project NEPTUNE, Grant ANR-17-CE09- 
0046-02).

[1] Salanne M, Rotenberg B, Naoi K, Kaneko K, Taberna PL, et al. 2016. Efficient Storage Mechanisms for Building Better Supercapacitors. Nat. Energy 1:16070

[2] Seh ZW, Kibsgaard J, Dickens CF, Chorkendorff I, Norskov JK, Jaramillo TF. 2017. Combining theory and experiment in electrocatalysis: Insights into materials design. Science 355

[3] Parsons R. 1990. The electrical double layer: recent experimental and theoretical developments. Chem. Rev. 90:813-826

[4] Bazant MZ, Storey BD, Kornyshev AA. 2011. Double layer in ionic liquids: Overscreening versus crowding. Phys. Rev. Lett. 106:046102

[5] McEldrew M, Goodwin ZAH, Kornyshev AA, Bazant MZ. 2018. Theory of the Double Layer in Water-in-Salt Electrolytes. J. Phys. Chem. Lett. 9:5840-5846

[6] Bazant MZ, Thornton K, Ajdari A. 2004. Diffuse-charge dynamics in electrochemical systems. Phys. Rev. E 70:021506

[7] Netz RR. 2003. Electrofriction and Dynamic Stern Layers at Planar Charged Surfaces. Phys. Rev. Lett. 91:138101

[8] Grün F, Jardat M, Turq P, Amatore C. 2004. Relaxation of the electrical double layer after an electron transfer approached by Brownian dynamics simulation. J. Chem. Phys. 120:9648

[9] Lobaskin V, Netz RR. 2016. Diffusive-convective transition in the non-equilibrium charging of an electric double layer. Europhys. Lett. 116:58001

[10] Asta AJ, Palaia I, Trizac E, Levesque M, Rotenberg B. 2019. Lattice Boltzmann electrokinetics simulation of nanocapacitors. J. Chem. Phys. 151:114104

[11] Striolo A, Michaelides A, Joly L. 2016. The carbon-water interface: Modeling challenges and opportunities for the waterenergy nexus. Annu. Rev. Chem. Biomol. Eng. 7:533-556

[12] Taylor CD, Wasileski SA, Filhol JS, Neurock M. 2006. First principles reaction modeling of the electrochemical interface: Consideration and calculation of a tunable surface potential from atomic and electronic structure. Phys. Rev. B 73:165402

[13] Lautar AK, Hagopian A, Filhol JS. 2020. Modeling interfacial electrochemistry: concepts and tools. Phys. Chem. Chem. Phys. 22:10569-10580

[14] Merlet C, Rotenberg B, Madden PA, Taberna PL, Simon P, et al. 2012. On the Molecular Origin of Supercapacitance in Nanoporous Carbon Electrodes. Nat. Mater. 11:306-310

[15] Simoncelli M, Ganfoud N, Sene A, Haefele M, Daffos B, et al. 2018. Blue energy and desalination with nanoporous carbon electrodes: Capacitance from molecular simulations to continuous models. Phys. Rev. X 8:021024

[16] Merlet C, Limmer DT, Salanne M, van Roij R, Madden PA, et al. 2014. The electric double layer has a life of its own. J. Phys. Chem. C 118:18291-18298

[17] Fedorov MV, Kornyshev AA. 2014. Ionic liquids at electrified interfaces. Chem. Rev. 114:2978-3036

[18] Burt R, Birkett G, Zhao XS. 2014. A review of molecular modelling of electric double layer capacitors. Phys. Chem. Chem. Phys. 16:6519-6538

[19] Torrie GM, Valleau JP, Patey GN. 1982. Electrical double layers. II. Monte Carlo and HNC studies of image effects. J. Chem. Phys. 76:4615-4622

[20] Glosli JN, Philpott MR. 1992. Molecular dynamics simulation of adsorption of ions from aqueous media onto charged electrodes. J. Chem. Phys. 96:6962-6969

[21] Kiyohara K, Asaka K. 2007. Monte Carlo simulation of electrolytes in the constant voltage ensemble. J. Chem. Phys. 126:214704

[22] Kiyohara K, Asaka K. 2007. Monte Carlo Simulation of Porous Electrodes in the Constant Voltage Ensemble. J. Phys. Chem. C 111:15903-15909

[23] van Megen W, Snook I. 1980. The grand canonical ensemble Monte Carlo method applied to the electrical double layer. J. Chem. Phys. 73:4656-4662

[24] Crozier PS, Rowley RL, Henderson D. 2001. Molecular-dynamics simulations of ion size effects on the fluid structure of aqueous electrolyte systems between charged model electrodes. J. Chem. Phys. 114:7513-7517

[25] Lee SH, Rasaiah JC, Hubbard JB. 1986. Molecular dynamics study of a dipolar fluid between charged plates. J. Chem. Phys. 85:5232-5237

[26] Hautman J, Halley JW, Rhee YJ. 1989. Molecular dynamics simulation of water beween two ideal classical metal walls. J. Chem. Phys. 91:467-472

[27] Rose DA, Benjamin I. 1993. Adsorption of $\mathrm{Na}^{+}$and $\mathrm{Cl}^{-}$at the charged water-platinum interface. J. Chem. Phys. 98:2283-2290

[28] Smith BB, Halley JW. 1994. Simulation study of the ferrous ferric electron transfer at a metal-aqueous electrolyte interface. J. Chem. Phys. 101:10915-10924

[29] Daub CD, Bratko D, Leung K, Luzar A. 2007. Electrowetting at the Nanoscale. J. Phys. Chem. C 111:505-509

[30] Siepmann JI, Sprik M. 1995. Influence of Surface-Topology and Electrostatic Potential on Water Electrode Systems. J. Chem. Phys. 102:511-524

[31] Reed SK, Lanning OJ, Madden PA. 2007. Electrochemical Interface Between an Ionic Liquid and a Model Metallic Electrode. J. Chem. Phys. 126:084704

[32] Pounds M, Tazi S, Salanne M, Madden PA. 2009. Ion adsorption at a metallic electrode: an ab initio based simulation 
study. J. Phys.: Condens. Matter 21:424109

[33] Vatamanu J, Borodin O, Smith GD. 2009. Molecular dynamics simulations of atomically flat and nanoporous electrodes with a molten salt electrolyte. Phys. Chem. Chem. Phys. 12:170-182

[34] Petersen MK, Kumar R, White HS, Voth GA. 2012. A computationally efficient treatment of polarizable electrochemical cells held at a constant potential. J. Phys. Chem. C 116:4903-4912

[35] Parsonage NG, Nicholson D. 1986. Computer simulation of water between metal walls. J. Chem. Soc., Faraday Trans. 2 82:1521-1535

[36] Gardner AA, Valleau JP. 1987. Water-like particles at surfaces. II. In a double layer and at a metallic surface. J. Chem. Phys. 86:4171-4176

[37] Takae K, Onuki A. 2015. Fluctuations of local electric field and dipole moments in water between metal walls. J. Chem. Phys. 143:154503

[38] Girotto M, dos Santos AP, Levin Y. 2017. Simulations of ionic liquids confined by metal electrodes using periodic Green functions. J. Chem. Phys. 147:074109

[39] Arnold A, Breitsprecher K, Fahrenberger F, Kesselheim S, Lenz O, Holm C. 2013. Efficient algorithms for electrostatic interactions including dielectric contrasts. Entropy 15:4569-4588

[40] Allen R, Hansen JP, Melchionna S. 2001. Electrostatic potential inside ionic solutions confined by dielectrics: a variational approach. Phys. Chem. Chem. Phys. 3:4177-4186

[41] Boda D, Gillespie D, Nonner W, Henderson D, Eisenberg B. 2004. Computing induced charges in inhomogeneous dielectric media: Application in a Monte Carlo simulation of complex ionic systems. Phys. Rev. E 69:046702

[42] Tyagi S, Süzen M, Sega M, Barbosa M, Kantorovich SS, Holm C. 2010. An iterative, fast, linear-scaling method for computing induced charges on arbitrary dielectric boundaries. J. Chem. Phys. 132:154112

[43] Breitsprecher K, Szuttor K, Holm C. 2015. Electrode models for ionic liquid-based capacitors. J. Phys. Chem. C 119:22445-22451

[44] Barros K, Sinkovits D, Luijten E. 2014. Efficient and accurate simulation of dynamic dielectric objects. J. Chem. Phys. 140:064903

[45] Geada IL, Ramezani-Dakhel H, Jamil T, Sulpizi M, Heinz H. 2018. Insight into induced charges at metal surfaces and biointerfaces using a polarizable Lennard-Jones potential. Nat. Commun. 9:716

[46] Iori F, Corni S. 2008. Including image charge effects in the molecular dynamics simulations of molecules on metal surfaces. J. Comput. Chem. 29:1656-1666

[47] Iori F, Felice RD, Molinari E, Corni S. 2009. GolP: An atomistic force-field to describe the interaction of proteins with $\mathrm{Au}(111)$ surfaces in water. J. Comput. Chem. 30:1465-1476

[48] Schmickler W, Henderson D. 1984. The interphase between jellium and a hard sphere electrolyte. A model for the electric double layer. J. Chem. Phys. 80:3381-3386

[49] Shelley JC, Patey GN, Bérard DR, Torrie GM. 1997. Modeling and structure of mercury-water interfaces. J. Chem. Phys. 107:2122-2141

[50] Price DL, Halley JW. 1995. Molecular dynamics, density functional theory of the metal-electrolyte interface. J. Chem. Phys. 102:6603-6612

[51] Walbran S, Mazzolo A, Halley J, Price D. 1998. Model for the electrostatic response of the copper - water interface. $J$. Chem. Phys. 109:8076-8080

[52] Finnis MW. 1991. The interaction of a point charge with an aluminium (111) surface. Surface Science 241:61-72

[53] Finnis MW, Kaschner R, Kruse C, Furthmuller J, Scheffler M. 1995. The interaction of a point charge with a metal surface: theory and calculations for (111), (100) and (110) aluminium surfaces. Journal of Physics: Condensed Matter 7:2001-2019

[54] Nalewajski RF. 1984. Electrostatic effects in interactions between hard (soft) acids and bases. J. Am. Chem. Soc. 106:944945

[55] Mortier WJ, Ghosh SK, Shankar S. 1986. Electronegativity-equalization method for the calculation of atomic charges in molecules. J. Am. Chem. Soc. 108:4315-4320

[56] Rappe AK, Goddard III WA. 1991. Charge equilibration for molecular dynamics simulations. J. Phys. Chem. 95:3358-3363

[57] York DM, Yang W. 1996. A chemical potential equalization method for molecular simulations. J. Chem. Phys. 104:159172. Publisher: American Institute of Physics

[58] Gingrich TR, Wilson M. 2010. On the ewald summation of gaussian charges for the simulation of metallic surfaces. Chem. Phys. Lett. 500:178-183

[59] Coretti A, Scalfi L, Bacon C, Rotenberg B, Vuilleumier R, et al. 2020. Mass-zero constrained molecular dynamics for electrode charges in simulations of electrochemical systems. J. Chem. Phys. 152:194701

[60] Streitz FH, Mintmire JW. 1994. Electrostatic potentials for metal-oxide surfaces and interfaces. Phys. Rev. B 50:1199612003

[61] Onofrio N, Strachan A. 2015. Voltage equilibration for reactive atomistic simulations of electrochemical processes. $J$. Chem. Phys. 143:054109

[62] Onofrio N, Guzman D, Strachan A. 2015. Atomic origin of ultrafast resistance switching in nanoscale electrometallization cells. Nat. Mater. 14:440-446

[63] Liang T, Antony AC, Akhade SA, Janik MJ, Sinnott SB. 2018. Applied Potentials in Variable-Charge Reactive Force Fields for Electrochemical Systems. J. Phys. Chem. A 122:631-638

[64] Nakano H, Sato H. 2019. A chemical potential equalization approach to constant potential polarizable electrodes for electrochemical-cell simulations. J. Chem. Phys. 151:164123 
[65] Buraschi M, Sansotta S, Zahn D. 2020. Polarization effects in dynamic interfaces of platinum electrodes and ionic liquid phases: A molecular dynamics study. J. Phys. Chem. C 124:2002-2007

[66] Nistor RA, Müser MH. 2009. Dielectric properties of solids in the regular and split-charge equilibration formalisms. Phys. Rev. B 79:104303

[67] Pastewka L, Järvi TT, Mayrhofer L, Moseler M. 2011. Charge-transfer model for carbonaceous electrodes in polar environments. Phys. Rev. B 83:165418

[68] Salanne M. 2015. Simulations of Room Temperature Ionic Liquids: from Polarizable to Coarse-Grained Force Fields. Phys. Chem. Chem. Phys. 17:14270-14279

[69] Merlet C, Salanne M, Rotenberg B, Madden PA. 2013. Influence of solvation on the structural and capacitive properties of electrical double layer capacitors. Electrochim. Acta 101:262-271

[70] Tazi S, Salanne M, Simon C, Turq P, Pounds M, Madden PA. 2010. Potential-induced ordering transition of the adsorbed layer at the ionic liquid / electrified metal interface. J. Phys. Chem. B 114:8453-8459

[71] Bedrov D, Piquemal JP, Borodin O, MacKerell, Jr. AD, Roux B, Schröder C. 2019. Molecular dynamics simulations of ionic liquids and electrolytes using polarizable force fields. Chem. Rev. 119:7940-7995

[72] Park S, McDaniel JG. 2020. Interference of electrical double layers: Confinement effects on structure, dynamics, and screening of ionic liquids. J. Chem. Phys. 152:074709

[73] Le Breton G, Joly L. 2020. Molecular modeling of aqueous electrolytes at interfaces: Effects of long-range dispersion forces and of ionic charge rescaling. J. Chem. Phys. 152:241102

[74] Spohr E, Heinzinger K. 1986. Molecular dynamics simulation of a water/metal interface. Chem. Phys. Lett. 123:218-221

[75] Spohr E. 1989. Computer simulation of the water/platinum interface. J. Phys. Chem. 93:6171-6180

[76] Al-Hamdani YS, Alfè D, Michaelides A. 2017. How strongly do hydrogen and water molecules stick to carbon nanomaterials? J. Chem. Phys. 146:094701

[77] Heinz H, Vaia RA, Farmer BL, Naik RR. 2008. Accurate simulation of surfaces and interfaces of face-centered cubic metals using 12-6 and 9-6 lennard-jones potentials. J. Phys. Chem. C 112:17281-17290

[78] Clabaut P, Fleurat-Lessard P, Michel C, Steinmann SN. 2020. Ten facets, one force field: The GAL19 force field for water-noble metal interfaces. J. Chem. Theory Comput. 16:4565-4578

[79] Marin-Laflèche A, Haefele M, Scalfi L, Coretti A, Dufils T, et al. 2020. MetalWalls: A Classical Molecular Dynamics Software Dedicated to the Simulation of Electrochemical Systems (doi: 10.26434/chemrxiv.12389777.v1)

[80] Some open source classical molecular dynamics simulation codes allowing the simulation of electrodes. Metalwalls: https://gitlab.com/ampere2/metalwalls; LAMMPS: https://lammps.sandia.gov for the distribution, https:// github.com/zhenxingwang/lamms-conp for the constant-potential fix; OpenMM: http://openmm.org for the distribution, https://github.com/scychon/openmm_constV for the constant-potential plug-in; ESPRESSO: espressomd.org.

[81] Yeh IC, Berkowitz ML. 1999. Ewald summation for systems with slab geometry. J. Chem. Phys. 111:3155-3162

[82] Dufils T, Jeanmairet G, Rotenberg B, Sprik M, Salanne M. 2019. Simulating Electrochemical Systems by Combining the Finite Field Method with a Constant Potential Electrode. Phys. Rev. Lett. 123:195501

[83] Stengel M, Spaldin NA, Vanderbilt D. 2009. Electric displacement as the fundamental variable in electronic-structure calculations. Nature Phys. 5:304-308

[84] Zhang C, Sprik M. 2016. Finite field methods for the supercell modeling of charged insulator/electrolyte interfaces. Phys. Rev. B 94:245309

[85] Sayer T, Zhang C, Sprik M. 2017. Charge compensation at the interface between the polar $\mathrm{NaCl}(111)$ surface and a $\mathrm{NaCl}$ aqueous solution. J. Chem. Phys. 147:104702

[86] Bonnet N, Morishita T, Sugino O, Otani M. 2012. First-Principles Molecular Dynamics at a Constant Electrode Potential. Phys. Rev. Lett. 109:266101

[87] Punnathanam SN. 2014. A Gibbs-ensemble based technique for Monte Carlo simulation of electric double layer capacitors (EDLC) at constant voltage. J. Chem. Phys. 140:174110

[88] Stenberg S, Stenqvist B, Woodward C, Forsman J. 2020. Grand canonical simulations of ions between charged conducting surfaces using exact 3D Ewald summations. Phys. Chem. Chem. Phys. 22:13659-13665

[89] Haskins JB, Lawson JW. 2016. Evaluation of molecular dynamics simulation methods for ionic liquid electric double layers. J. Chem. Phys. 144:134701

[90] Scalfi L, Limmer DT, Coretti A, Bonella S, Madden PA, et al. 2020. Charge fluctuations from molecular simulations in the constant-potential ensemble. Phys. Chem. Chem. Phys. 22:10480-10489

[91] Limmer DT, Merlet C, Salanne M, Chandler D, Madden PA, et al. 2013. Charge fluctuations in nanoscale capacitors. Phys. Rev. Lett. 111:106102

[92] Takahashi K, Nakano H, Sato H. 2020. A polarizable molecular dynamics method for electrode-electrolyte interfacial electron transfer under the constant chemical-potential-difference condition on the electrode electrons. J. Chem. Phys. 153:054126

[93] Kattirtzi JA, Limmer DT, Willard AP. 2017. Microscopic dynamics of charge separation at the aqueous electrochemical interface. Proc. Natl. Acad. Sci. U.S.A. 114:13374-13379

[94] Willard AP, Reed SK, Madden PA, Chandler D. 2009. Water at an electrochemical interface - a simulation study. Faraday Discuss. 141:423-441

[95] Bonthuis DJ, Gekle S, Netz RR. 2012. Profile of the Static Permittivity Tensor of Water at Interfaces: Consequences for Capacitance, Hydration Interaction and Ion Adsorption. Langmuir 28:7679-7694

[96] Jeanmairet G, Rotenberg B, Borgis D, Salanne M. 2019. Study of a water-graphene capacitor with molecular density functional theory. J. Chem. Phys. 151:124111 
[97] Li Z, Jeanmairet G, Mendez-Morales T, Rotenberg B, Salanne M. 2018. Capacitive Performance of Water-in-Salt Electrolytes in Supercapacitors: A Simulation Study. J. Phys. Chem. C 122:23917-23924

[98] Suo L, Hu YS, Li H, Armand M, Chen L. 2013. A New Class of Solvent-in-Salt Electrolyte for High-Energy Rechargeable Metallic Lithium Batteries. Nat. Commun. 4:1481

[99] Wang J, Yamada Y, Sodeyama K, Chiang CH, Tateyama Y, Yamada A. 2016. Superconcentrated Electrolytes for a High-voltage Lithium-ion Battery. Nat. Commun. 7:12032

[100] Rotenberg B, Salanne M. 2015. Structural transitions at ionic liquid interfaces. J. Phys. Chem. Lett. 6:4978-4985

[101] Uralcan B, Aksay IA, Debenedetti PG, Limmer DT. 2016. Concentration Fluctuations and Capacitive Response in Dense Ionic Solutions. J. Phys. Chem. Letters 7:2333-2338

[102] Péan C, Merlet C, Rotenberg B, Madden PA, Taberna PL, et al. 2014. On the dynamics of charging in nanoporous carbon-based supercapacitors. ACS Nano 8:1576-1583

[103] Pean C, Daffos B, Rotenberg B, Levitz P, Haefele M, et al. 2015. Confinement, desolvation, and electrosorption effects on the diffusion of ions in nanoporous carbon electrodes. J. Am. Chem. Soc. 137:12627

[104] Pean C, Rotenberg B, Simon P, Salanne M. 2016. Multi-scale modelling of supercapacitors: From molecular simulations to a transmission line model. J. Power Sources 326:680-685

[105] Breitsprecher K, Holm C, Kondrat S. 2018. Charge Me Slowly, I Am in a Hurry: Optimizing Charge/Discharge Cycles in Nanoporous Supercapacitors. ACS Nano 12:9733-9741

[106] Limmer DT, Willard AP, Madden P, Chandler D. 2013. Hydration of metal surfaces can be dynamically heterogeneous and hydrophobic. Proc. Natl. Acad. Sci. U.S.A. 110:4200-4205

[107] Mullen RG, Shea JE, Peters B. 2014. Transmission Coefficients, Committors, and Solvent Coordinates in Ion-Pair Dissociation. J. Chem. Theory Comput. 10:659-667

[108] Willard AP, Limmer DT, Madden PA, Chandler D. 2013. Characterizing heterogeneous dynamics at hydrated electrode surfaces. J. Chem. Phys. 138:184702

[109] Limmer DT, Willard AP, Madden PA, Chandler D. 2015. Water Exchange at a Hydrated Platinum Electrode is Rare and Collective. J. Phys. Chem. C 119:24016-24024

[110] Limmer DT, Willard AP. 2015. Nanoscale heterogeneity at the aqueous electrolyte-electrode interface. Chem. Phys. Lett. 620:144-150

[111] Zhang Y, Stirnemann G, Hynes JT, Laage D. 2020. Water dynamics at electrified graphene interfaces: a jump model perspective. Phys. Chem. Chem. Phys. 22:10581-10591

[112] Marcus RA. 1956. On the theory of oxidation-reduction reactions involving electron transfer. I. J. Chem. Phys. 24:966-978

[113] Warshel A. 1982. Dynamics of reactions in polar solvents. semiclassical trajectory studies of electron-transfer and protontransfer reactions. J. Phys. Chem. 86:2218-2224

[114] Reed SK, Madden PA, Papadopoulos A. 2008. Electrochemical charge transfer at a metallic electrode: a simulation study. J. Chem. Phys. 128:124701

[115] Pounds MA, Salanne M, Madden PA. 2015. Molecular aspects of the $\mathrm{Eu}^{3+} / \mathrm{Eu}^{2+}$ redox reaction at the interface between a molten salt and a metallic electrode. Mol. Phys. 113:2451-2462

[116] Wilhelm F, Schmickler W, Nazmutdinov R, Spohr E. 2011. Modeling proton transfer to charged silver electrodes. Electrochimica Acta 56:10632-10644

[117] Wiebe J, Spohr E. 2017. Water Structure and Mechanisms of Proton Discharge on Platinum Electrodes: Empirical Valence Bond Molecular Dynamics Trajectory Studies. Electrocatalysis 8:637-646

[118] Dwelle KA, Willard AP. 2019. Constant potential, electrochemically active boundary conditions for electrochemical simulation. J. Phys. Chem. C 123:24095-24103

[119] Rose DA, Benjamin I. 1991. Solvation of $\mathrm{Na}^{+}$and $\mathrm{Cl}^{-}$at the water-platinum (100) interface. J. Chem. Phys. 95:6856-6865

[120] Calhoun A, Voth GA. 1996. Electron Transfer Across the Electrode/Electrolyte Interface: Influence of Redox Ion Mobility and Counterions. J. Phys. Chem. 100:10746-10753

[121] Scalfi L, Dufils T, Reeves K, Rotenberg B, Salanne M. 2020. A semiclassical Thomas-Fermi model to tune the metallicity of electrodes in molecular simulations. J. Chem. Phys. 153:174704

[122] Schlaich A, Jin D, Bocquet L, Coasne B. 2020. Wetting transition of ionic liquids at metal surfaces: A computational approach to electronic screening using a virtual Thomas-Fermi fluid. https://arxiv.org/abs/2002.11526

[123] Comtet J, Niguès A, Kaiser V, Coasne B, Bocquet L, Siria A. 2017. Nanoscale capillary freezing of ionic liquids confined between metallic interfaces and the role of electronic screening. Nat. Mater. 16:634-639

[124] Jeanmairet G, Rotenberg B, Levesque M, Borgis D, Salanne M. 2019. A molecular density functional theory approach to electron transfer reactions. Chem. Sci. 10:2130-2143

[125] Fajardo OY, Bresme F, Kornyshev AA, Urbakh M. 2015. Electrotunable lubricity with ionic liquid nanoscale films. Sci. Rep. 5:7698 


\section{Supplemental Material for: \\ Molecular Simulation \\ of Electrode-Solution Interfaces}

Laura Scalfi, Mathieu Salanne, Benjamin Rotenberg

(Dated: Aug. 2020)

PACS numbers:

\section{CAPACITANCE}

(a)

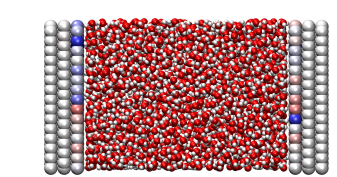

(b)

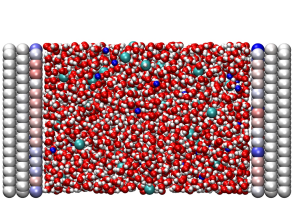

$q(e)$

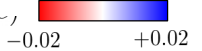

(c)

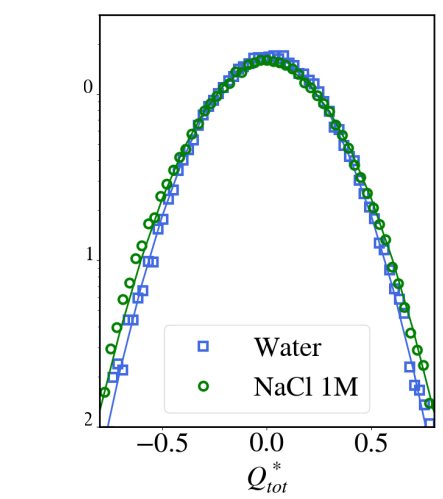

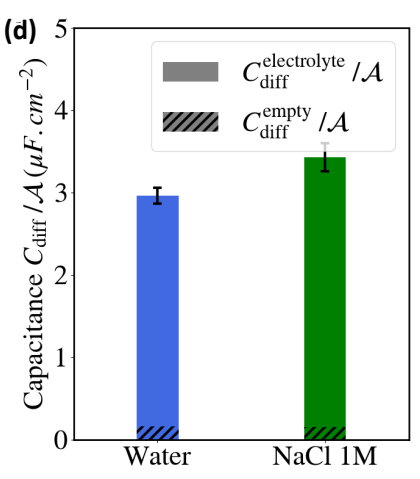

FIG. 1: Capacitors consisting of graphite electrodes separated by (a) pure water and (b) a $1 \mathrm{M}$ aqueous NaCl solution under a voltage $\Delta \Psi=0 \mathrm{~V}$; the color bar indicates the instantaneous charge of the electrode atoms. (c) Distribution of the total charge of the electrode $Q_{\text {tot }}^{*}$ for both systems, computed within Born-Oppenheimer dynamics simulations; solid lines are normalized Gaussian distributions with the corresponding standard deviation. (d) Contributions to the differential capacitance per unit area $C_{\text {diff }} / \mathcal{A}$ at $0 \mathrm{~V}$ : from the empty capacitor, $C_{\text {diff }}^{\text {empty }}$, and from the charge fluctuations induced by the electrolyte, $C_{\text {diff }}^{\text {electrolyte }}=\beta\left\langle\delta Q_{t o t}^{* 2}\right\rangle$ (see Eq. 5 of the main text); the latter corresponds to the variance of the distributions of panel (c). Panels a, b and d adapted from Ref. [1], Phys. Chem. Chem. Phys., 2020, 22, 10480 by permission of the PCCP Owner Societies.

Supplementary Figure 1 illustrates the discussion of Section 4.1 of the main text with results from Scalfi et al. for two capacitors consisting of graphite electrodes separated by pure water or a $1 \mathrm{M}$ aqueous $\mathrm{NaCl}$ solution, under a voltage $\Delta \Psi=0 \mathrm{~V}[1]$. The variance of the distribution $P\left(Q_{t o t}^{*}\right)$ of the total charge computed in Born-Oppenheimer dynamics simulation in the constant-potential ensemble (see Section 3.2.2), provides the contribution of the electrolyte, $C_{\text {diff }}^{\text {eletrolyte }}=\beta\left\langle\delta Q_{t o t}^{* 2}\right\rangle$, to the differential capacitance. This contribution is large compared to that of the empty capacitor, $C_{\text {diff }}^{\text {empty }}$, consistently with the observation of Haskins and Lawson for ionic liquids [2] - and with the fact that it had escaped the attention of the community until recently.

\section{ELECTROWETTING}

Electrochemistry also goes beyond simple setups with two electrodes facing each other and an electrolyte in between. For example, electrowetting-on-dielectric setups were proposed for switchable optical devices. An example of design is made of two concentric electrodes (with different radii) separated from the liquid, which is made of a water nanodrop, by a layer of dielectric [4]. The use of constant potential simulations allowed an accurate representation of such systems at the molecular scale [3], as shown on Figure 2a. Applying a potential between the two electrodes results in a noticeable spreading of the drop, an effect which is reversible. Figure $2 \mathrm{~b}$ shows the cross-section profiles at various applied potentials, from which contact angles can be extracted. In this example the angle varies from $111^{\circ}$ at null voltage down to $84^{\circ}$ at $4 \mathrm{~V}$. Changing the radii of the two electrodes leads to different values but the spreading effect persists. At the macroscale, the variation of the contact angle with the applied potential is well predicted by the Young-Lippmann equation, but simulations showed that it does not hold anymore at the nanometric scales. 
(a)

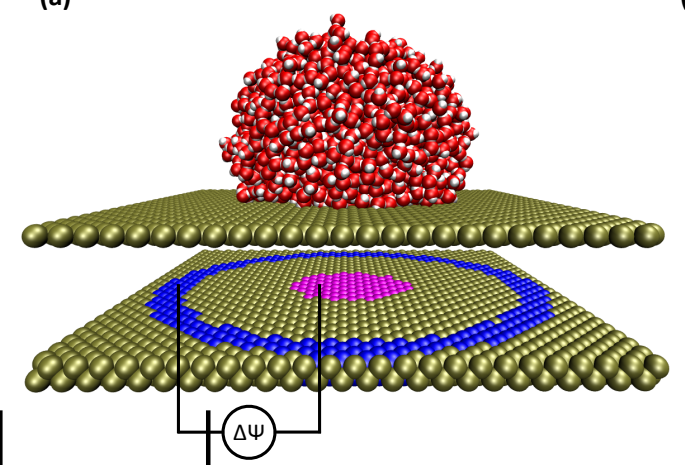

(b)

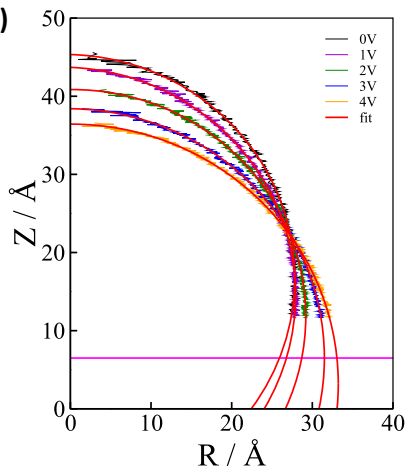

FIG. 2: (a) Representation of the electrowetting-on-dielectric simulated system. Ochre atoms below the aqueous drop constitute a hydrophobic insulator monolayer. The magenta and blue atoms are the inner and outer platinum electrodes, which are also separated by an insulator ring (a vertical shift was introduced between the two layers of the substrate material for a better visualization). (b) Vertical cross section of the water droplet on the insulator surface at various applied voltages. The contact angle is determined by a fit of the drop contour. Adapted with permission from Ref. [3], ACS Nano 2016, 10, 8536, Copyright (2016) American Chemical Society.

By performing additional non-equilibrium simulations where the potential was suddenly applied or released, it was observed that the retraction at zero voltage is much faster than the corresponding spreading, an effect which could be explained by different liquid/solid friction properties.

[1] L. Scalfi, D. T. Limmer, A. Coretti, S. Bonella, P. A. Madden, M. Salanne, and B. Rotenberg. Charge fluctuations from molecular simulations in the constant-potential ensemble. Phys. Chem. Chem. Phys., 22:10480-10489, 2020.

[2] J. B. Haskins and J. W. Lawson. Evaluation of molecular dynamics simulation methods for ionic liquid electric double layers. J. Chem. Phys., 144:134701, 2016.

[3] J. R. Choudhuri, D. Vanzo, P. A. Madden, M. Salanne, D. Bratko, and A. Luzar. Dynamic response in nanoelectrowetting on a dielectric. ACS Nano, 10:8536-8544, 2016.

[4] T. Krupenkin, S. Yang, and P. Mach. Tunable liquid microlens. Appl. Phys. Lett., 82:316, 2003. 